CASE REPORT

\title{
Hematemesis Following Rapid Recanalization of the Obstructed Left Main Bronchus with Crizotinib in a 92-year-old Patient with EML4-ALK Rearrangement-positive Lung Adenocarcinoma
}

\author{
Takashi Iwata1; Michihito Toda1; Naomi Ohta2; \\ Masaaki Izumi2; Shoji Hanadal; Takuya Miural
}

complete recanalization of left main bronchus. In addition, the liver tumor had significantly decreased in size on $\mathrm{CT}$. The patient did not require further oxygen inhalation; however, she refused to take crizotinib on day 8 due to repeated diarrhea and constipation resulting in frequent abdominal pain. Unfortunately, a fever developed six days after crizotinib cessation, and the patient suddenly died two days later of massive hematemesis. An autopsy was not performed, and the exact cause of hematemesis was unknown. Conclusions. Crizotinib has a substantial anticancer effect, even in nonagenarians. To the best of our knowledge, the current patient is the oldest to exhibit a dramatic and rapid response to crizotinib. The relationship between sudden death due to hematemesis and the administration of crizotinib is unknown. The patient's condition in this case may have simply involved the acute exacerbation of preexisting hemorrhagic gastritis, although the rapid deterioration of undetected intestinal metastatic lesions is also possible.

(JJLC. 2014;54:951-954)

KEY WORDS — Non-small cell lung cancer, Crizotinib, Aged, Hematemesis

\section{CASE}

A 92-year-old woman with chronic renal dysfunction presented with a low-grade fever and dry cough lasting for one month and was admitted to the Department of Internal Medicine at our hospital. A chest radiograph showed left total atelectasis (Figure 1A), and computed tomography (CT) demonstrated a left hilar mass totally obstructing the main bronchus (Figure 1B). Multiple low-density areas were also noted in the liver (Figure $1 \mathrm{C}$ ), and fiberoptic gastroscopy revealed a hiatal hernia with slight hemorrhagic gastritis. Therefore, the admini-

\footnotetext{
1Department of Thoracic and Cardiovascular Surgery, ${ }^{2}$ Department of Internal Medicine, Kansai Rosai Hospital, Japan.

Reprints: Takashi Iwata, Department of Thoracic and Cardio-
}

vascular Surgery, Kansai Rosai Hospital, 3-1-69 Inabaso, Amagasaki, Hyogo 660-8511, Japan (e-mail: taiwata@kanrou.net).

Received August 6, 2014; accepted September 19, 2014. 


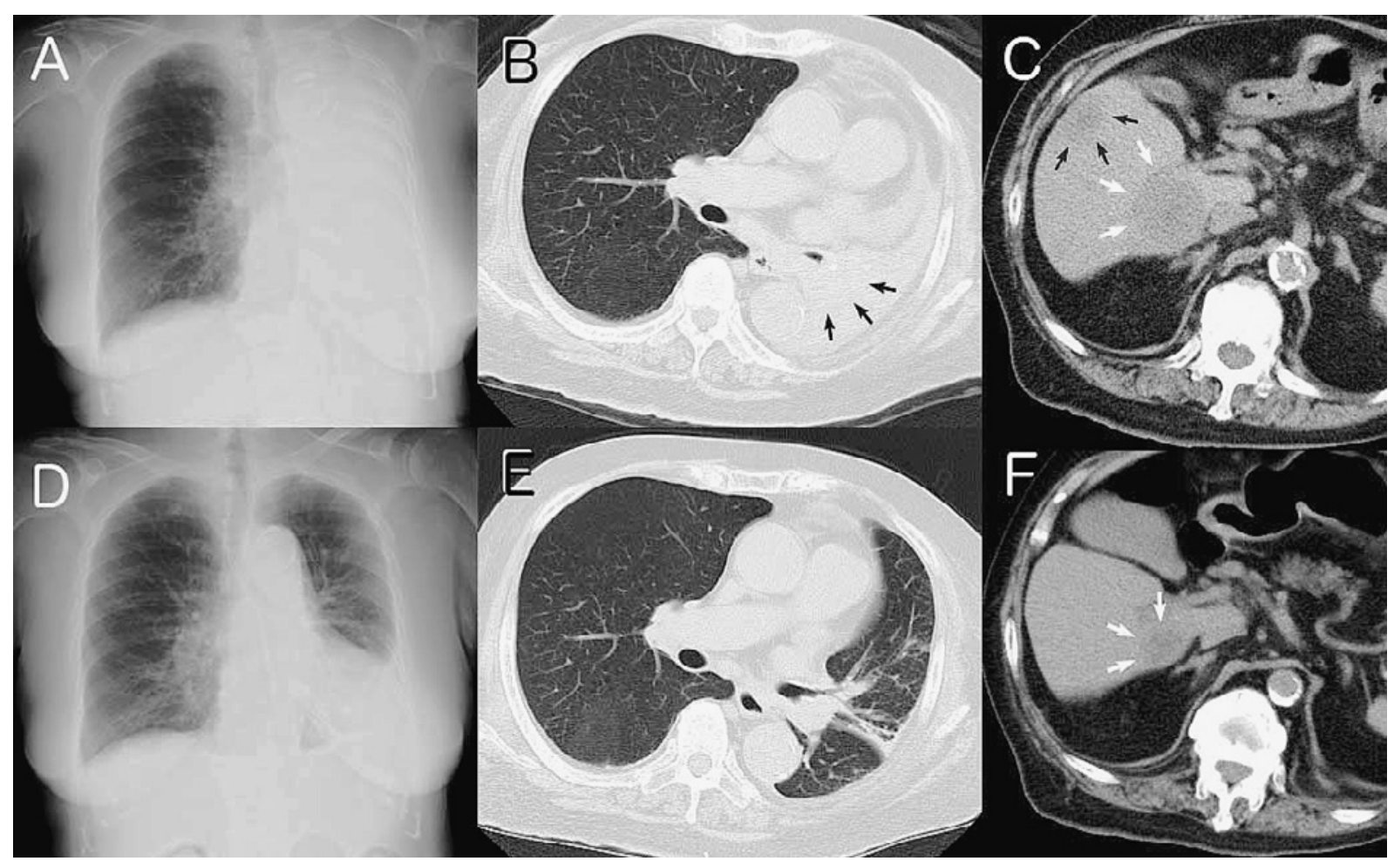

Figure 1. A. Chest radiography shows total atelectasis of the left lung. B. Chest computed tomography (CT) demonstrates a left hilar mass obstructing the left main bronchus (arrows). C. Abdominal CT discloses multiple metastatic lesions in the liver (white and black arrows). D. Chest radiography performed on day 5 after crizotinib induction shows total expansion of the left lung. E. Chest CT performed on day 13 demonstrates recanalization of the left main bronchus. F. Abdominal CT conducted on the same day also shows shrinkage of the liver lesion (arrows).

stration of a proton-pump inhibitor was started after the examination. A needle biopsy of the liver disclosed poorly-differentiated adenocarcinoma with immunohistochemical staining positive for TTF-1. Therefore, left lung cancer with multiple liver metastases was diagnosed. Positron emission tomography was not performed. However, rearrangement of the echinoderm microtubule-associated protein-like 4-anaplastic lymphoma kinase (EML4-ALK) fusion gene was detected on both immunohistochemical staining and a FISH assay of the liver specimen. The patient refused any further evaluations or treatment and was discharged. Eleven days later, she was again transferred to our department due to sudden hemoptysis. Upon arrival, no airway bleeding was observed, and her respiratory status was stable. The peripheral capillary oxygen saturation was $90 \%$ on room air; hence, oxygen $(1 \mathrm{l} / \mathrm{min})$ was administered via a nasal mask. The patient's performance status (ECOG) was 4. Fiberoptic bronchoscopy disclosed total obstruction of the left main bronchus by the ulcerative tumor. Bronchial artery embolization was thus under- taken; however, the responsible artery was not detected on angiography. Ten days later, treatment with crizotinib was started at a dose of $400 \mathrm{mg}$ per day. On day 5 , she experienced a drastic improvement in dyspnea, and a chest radiograph and CT scan showed complete recanalization of the left main bronchus (Figure 1D, 1E). The liver tumor had also drastically decreased in size on CT (Figure $1 \mathrm{~F}$ ), and she no longer required oxygen inhalation. However, she refused to take crizotinib on day 8 due to repeated diarrhea and constipation resulting in frequent abdominal pain. Unfortunately, a fever developed six days after crizotinib cessation, and, although a series of chest radiographs and abdominal CT scans showed no niveau or free air, the patient suddenly died of massive hematemesis two days later. An autopsy was not performed, and the exact cause of hematemesis remained unknown.

\section{DISCUSSION}

Gefitinib is now widely used and becoming accepted as a first-line treatment in elderly patients with epidermal 
growth factor receptor-mutated advanced lung adenocarcinoma due to its safety and efficacy. ${ }^{1,2}$ However, the feasibility and efficacy of crizotinib in elderly patients with advanced lung cancer exhibiting EML4-ALK fusion gene rearrangement have not been clarified. The oldest reported patient to receive gefitinib therapy in the literature was 94 years old, ${ }^{2}$ compared to 86 years for crizotinib. ${ }^{3}$ To the best of our knowledge, the current patient is the oldest to show a dramatic and rapid response to crizotinib.

The dose of crizotinib is another issue in the treatment of elderly subjects. Although the standard dose of crizotinib is $500 \mathrm{mg}$ /day, we chose to administer 400 $\mathrm{mg} /$ day as the initial dose in the present case because we believed that $400 \mathrm{mg}$ would be a safer dose due to the patient's age and impaired performance status.

The relationship between sudden death from hematemesis and treatment with crizotinib is unknown. Our patient's condition appeared to involve the acute exacerbation of preexisting hemorrhagic gastritis, although this may be unlikely because she had received a protonpump inhibitor for approximately two months. Another possibility is that crizotinib had some unknown adverse effect on her gastrointestinal system, or perhaps this complication is only observed in elderly patients. Two cases of esophageal inflammation induced by crizotinib have been reported in the literature. ${ }^{4,5}$ In the current case, the acute development of esophageal hemorrhagic ulcers cannot be strictly excluded. Although the patient had complained of repeated constipation and diarrhea causing frequent abdominal pain prior to the onset of sudden hematemesis, no symptoms suggestive of an esophageal lesion, such as swallowing pain or difficulty, were observed throughout her entire clinical course. Therefore, we consider enterocolonic lesions to most likely be responsible for the hematemesis. For example, the rapid deterioration of undetected intestinal metastatic lesion(s) induced by crizotinib is a possibility. One case of perforation of a metastatic tumor in the stomach in a patient who received gefitinib has been reported in the literature. ${ }^{6}$ Crizotinib therapy may have significant anti-tumor effects, even in elderly patients, with rapid recanalization of the main bronchus and rapid relief of dyspnea, as in the present case. On the other hand, the rapid worsening of various metastatic sites may result in site-related organ failure and an associated severe critical condition. We inferred that crizotinib may have caused the rapid decay of undiagnosed mesenteric or intestinal metastases, making the intestinal wall partially fragile, in this case. Crizotinib is also known to cause functional motility disorders of the gastrointestinal tract. Excess movement and/or a high intraluminal pressure at fragile sites in the intestines may cause perforation, resulting in massive hematemesis and acute death. The potential for intestinal perforation is possible in all patients with intestinal metastasis treated with "effective" chemotherapy and is specific to neither crizotinib nor elderly patients.

A discussion regarding the safety of crizotinib in elderly patients in their eighties and nineties would be interesting. However, we found no such findings on this issue in the previous literature. Furthermore, administering cytotoxic agents in elderly patients with advanced lung cancer is very difficult, especially if the patient's performance status is impaired. Molecular-targeted agents, such as crizotinib, are sometimes safe and useful in these cases and may be a potential treatment option. The risks of crizotinib treatment in elderly patients with an impaired performance status remain unknown but worth considering. Special care should be taken when providing crizotinib therapy in elderly patients with an impaired status, as there is currently insufficient experience with crizotinib in this population.

本論文内容に関連する著者の利益相反：なし

\section{REFERENCES}

1. Maemondo M, Minegishi Y, Inoue A, Kobayashi K, Harada M, Okinaga S, et al. First-line gefitinib in patients aged 75 or older with advanced non-small cell lung cancer harboring epidermal growth factor receptor mutations: NEJ 003 study. J Thorac Oncol. 2012;7:1417-1422.

2. Tateishi K, Ichiyama T, Hirai K, Agatsuma T, Koyama S, Hachiya $\mathrm{T}$, et al. Clinical outcomes in elderly patients administered gefitinib as first-line treatment in epidermal growth factor receptor-mutated non-small-cell lung cancer: retrospective analysis in a Nagano Lung Cancer Research Group study. Med Oncol. 2013;30:450.

3. Camidge DR, Bang YJ, Kwak EL, Iafrate AJ, Varella-Garcia M, Fox SB, et al. Activity and safety of crizotinib in patients with ALK-positive non-small-cell lung cancer: updated results from a phase 1 study. Lancet Oncol. 2012;13:1011-1019.

4. Park J, Yoshida K, Kondo C, Shimizu J, Horio Y, Hijioka S, et al. Crizotinib-induced esophageal ulceration: a novel adverse event of crizotinib. Lung Cancer. 2013;81:495-496.

5. Srivastava N, VanderLaan PA, Kelly CP, Costa DB. Esophagitis: a novel adverse event of crizotinib in a patient with ALK-positive non-small-cell lung cancer. J Tho- 
rac Oncol. 2013;8:e23-e24.

6. Chung WP, Song HL, Ho CL, Chiu NT, Su WC. Gastric perforation secondary to regression of lung adenocarcinoma after gefitinib treatment. J Clin Oncol. 2013;31:e6-e8. 